\title{
An integrated in silico immuno-genetic analytical platform provides insights into COVID-19 serological and vaccine targets
}

\author{
Daniel Ward ${ }^{1 *} \mathbb{D}$, Matthew Higgins', Jody E. Phelan ${ }^{1}$, Martin L. Hibberd ${ }^{1}$, Susana Campino ${ }^{1}$ and Taane G. Clark ${ }^{1,2^{*}}$
}

\begin{abstract}
During COVID-19, diagnostic serological tools and vaccines have been developed. To inform control activities in a postvaccine surveillance setting, we have developed an online "immuno-analytics" resource that combines epitope, sequence, protein and SARS-CoV-2 mutation analysis. SARS-CoV-2 spike and nucleocapsid proteins are both vaccine and serological diagnostic targets. Using the tool, the nucleocapsid protein appears to be a sub-optimal target for use in serological platforms. Spike D614G (and nsp12 L314P) mutations were most frequent (> 86\%), whilst spike A222V/ $\mathrm{L} 18 \mathrm{~F}$ have recently increased. Also, Orf3a proteins may be a suitable target for serology. The tool can accessed from: http://genomics.Ishtm.ac.uk/immuno (online); https://github.com/dan-ward-bio/COVID-immunoanalytics (source code).
\end{abstract}

Keywords: SARS-CoV-2, COVID, Human-coronavirus, Immuno-informatics, Mutation, Epitopes, Cross-reactivity, Surveillance

\section{Background}

COVID-19, the disease caused by the SARS-CoV-2 virus, was first characterised in the city of Wuhan, Hubei, and has now spread to 190 countries. With over 60 million confirmed cases worldwide and more than 1.26 million deaths, the COVID-19 pandemic has placed a high burden on the world's healthcare infrastructure and economies, with projected final costs of 28 trillion or $31 \%$ of the global gross domestic product $[1,2]$. The majority of infections are either asymptomatic or result in mild flulike symptoms, with severe cases of viral pneumonia affecting between $1.0 \%$ ( $\geq 20$ years) and $18.4 \%$ ( $\geq 80$ years) of diagnosed patients [3]. Its variable infection outcome, mode of transmission and incubation period together have enhanced the ability of the pathogen to spread efficiently worldwide. As a result, there has been an urgent

\footnotetext{
* Correspondence: Daniel.ward1@lshtm.ac.uk; Taane.clark@lshtm.ac.uk 'Department of Infection Biology, Faculty of Infectious and Tropical Diseases, London School of Hygiene and Tropical Medicine, Keppel Street, London WC1E 7HT, UK

Full list of author information is available at the end of the article
}

push for the development of diagnostics, therapeutics and vaccines to aid control efforts.

Current front-line diagnostic strategies apply a quantitative reverse transcription PCR (RT-qPCR) assay on patient nasopharyngeal swabs, using primer/probe sets targeting the $n s p 10, R d R p$, nsp 14, envelope and nucleocapsid genes; tests endorsed by a number of agencies and health systems $[4,5]$. Patients hospitalised with severe respiratory disease who are RT-qPCR negative may be diagnosed radiographically (chest $\mathrm{x}$-ray or computerised tomography scan), but in limited resource or high infection rate settings, these methods maybe unviable. Considering the inherent limitations in the sample collection process and transient viral load, RNA detection-based diagnostics can vary in their sensitivity. The demand for serological diagnostics is high, particularly because these tests are capable of detecting SARSCoV-2 antibodies, which are biomarkers indicative of current infection that remains present after viral clearance [6]. These tools are essential to address crucial sero-epidemiological questions, like understanding viral 
prevalence across a population, the percentage of asymptomatic patients and longevity of antibody responses post infection.

Numerous lateral flow rapid diagnostic tests (RDTs) and enzyme-linked immunosorbent assays (ELISA) tests have been developed, including an approved IgM/IgG RDT which uses the nucleocapsid protein as a target for the detection of seroconverted individuals [7]. Other assays use the spike protein as an antigen, with some using the receptor-binding domain (RBD) as a target, a region with a high level of diversity between alphacoronavirus species [8]. Unlike RNA detection methods, these platforms can identify convalescent patients, which further informs outbreak control efforts.

Long-term control strategies will involve vaccine rollout. As of November 2020, there were more than 50 vaccines at development phase 1 or greater, with at least 10 vaccines in phase $3[9,10]$. Vaccines at the forefront include those based on a non-replicating adenovirus vector base (ChAdOx-nCoV-19 and Ad5-nCov), an LNPencapsulated mRNA (BNT162 and mRNA-1273), protein subunit (NVX-CoV2373) or inactivated virus (BBIBP-CorV and CoronaVac) [11]. The discovery, development and management of efficacious vaccines, as well as sensitive and specific serological diagnostics, are both dependant on the availability of up-to-date information on viral evolution and immune-informatic analyses. The identification of variable or conserved regions in the proteome of SARS-CoV-2 can inform the rational selection of reverse-design targets in both vaccinology and diagnostic fields, as well as indicate immunologically relevant regions of interest for further studies to characterise SARS-CoV-2 immune responses. Whilst the availability of biological data for SARS-CoV-2 in the public domain has increased, insights are most likely to come from its integration informatically in an open and accessible format.

\section{Construction and content \\ Rationale}

Here, we present an online integrated immuno-analytic resource for the visualisation and extraction of SARSCoV-2 meta-analysis data [12]. This website was built around an automated pipeline for the formation of a whole genome sequence based variant database for SARS-CoV-2 isolates worldwide (as of November 2020, $n=150,090$ ). We have integrated this dataset with a suite of B cell epitope prediction platform meta analyses, HLA-I and HLA-II peptide prediction, an 'epitope mapping' analysis of available experimental in vitro confirmed epitope data from the Immune Epitope Database (IEDB) [13] and a protein orthologue sequence analysis of six relevant coronavirus species (SARS, MERS, OC43, HKU1, NL63 and 229E), with all data updated and annotated regularly with information from the UniProt database. Additional functionality enables users to visualise external analytical datasets presented in the literature (e.g. [14]). Moreover, we have added functionally to spatio-temporally track non-synonymous mutations of interest through the dataset, allowing up-to-date surveillance of mutations that may be of immunological relevance. With this resource, users can browse the annotated SARS-CoV-2 proteome and extract meta data to inform further research and analyses.

\section{Whole genome sequence data analysis}

SARS-CoV-2 nucleotide sequences were downloaded from NCBI (https://www.ncbi.nlm.nih.gov) and GISAID (https://www.gisaid.org). As part of an automated inhouse pipeline, sequences were aligned using MAFFT software (v7.2) [15] and trimmed to the beginning of the first reading frame (orf1ab-nsp1). Sequences with $>20 \%$ missing were excluded from the dataset. Using data available from the NCBI COVID-19 resource, a modified annotation (GFF) file was generated and open reading frames (ORFs) for each respective viral protein were extracted (taking in to account ribosomal slippage) using the bedtools 'getfasta' function [16]. Each ORF was translated using EMBOSS transeq software [17], and the variants for each protein sequence were identified using an in-house script [18]. As a part of our analysis pipeline, we generated consensus sequences for each SARS$\mathrm{CoV}-2$ protein from the nucleotide database using the EMBOSS Cons CLI tool [17]. These canonical sequences were used as a reference for prediction, specificity and epitope mapping analyses.

\section{B cell epitope prediction meta-analysis}

Six epitope prediction software platforms were chosen for this analysis (Bepipred [19], AAPpred [20] DRREP [21], ABCpred [22], LBtope [23] and BCEpreds [24]). For each tool, we used the settings and quality cut-offs as recommended by their respective authors. The scores across the predictive platforms were then normalised (minimum-maximum scaled) to ensure that no single tool skewed the aggregate 'consensus' score, and combined to provide a single consensus B cell epitope prediction score. Within the 'raw data table' (accessed from the tool's landing page), users can dissect each score depending on their preference of methodology.

\section{HLA-I and HLA-II peptide prediction}

We have incorporated an HLA-I peptide prediction analysis within the tool to aid in the scrutiny and development of vaccine candidates. $\mathrm{CD}^{+}$effector immunity has been reported to play a central role in the response to SARS-CoV infection, as well as infection mediated immunopathology [25-27]. We used a database of 2915 
HLA-A, HLA-B and HLA-C alleles to make HLA-I peptide binding predictions using the netMHCpan server (v4.1) [28], with peptide lengths of 8 to 14 amino acids across the entire SARS-CoV-2 proteome. We chose to use the netMHCpan server for our HLA-I peptide prediction analysis, due to its high overall performance and its extensive HLA-I allele database [28]. We ran predictions for a total of 2915 alleles (HLA-A 886, HLA-B 1412 and HLA-C 617) across all peptide lengths (8-14 amino acids). The analysis generated 1.1 billion candidates. After quality control, we selected a total of 736, 073 peptides based on strong binding affinity across the allele database. We selected strong binding affinity peptides based on the tools internal binding scoring metrics. Only 'strong binding' alleles were selected for further analysis. For each position with a ligand with high binding affinity, we analysed the percentage representation of the respective HLA-I type across the allele database. For predicting HLA-II peptides we used the MARIA online tool [29]. We pre-processed the SARS-CoV-2 canonical protein sequences using a 15 amino acid sliding window. Predictions were made for all available HLA-II alleles. A 95\% cut off was chosen for a positive HLA-II presentation. All data for each 15-mer is displayed on the tool.

\section{Epitope mapping}

B cell epitopes for coronavirus species were sourced from the Immune Epitope Database (IEDB) resource (https://www.iedb.org, updated: October 2020) [13]. Using BLASTp [30], we mapped short amino acid epitope sequences onto the canonical sequence of SARS$\mathrm{CoV}-2$ proteins. A BLASTp bitscore of 25 with a minimum length of 8 residues was selected as a quality cutoff for mapped epitopes. The frequency of mapped epitopes was logged for each position in the protein and parsed for graphical representation.

\section{Coronavirus homology analysis}

Reference proteomes for SARS, MERS, OC43, 229E, HKU1 and NL63 $\alpha$ and $\beta$ coronavirus (-CoV) species were sourced from UniProt database. These sequences were processed into 10-mers using the pyfasta platform and mapped on to the canonical sequences of SARS$\mathrm{CoV}-2$ proteins using the aforementioned 'epitope mapping' process. The $\mathrm{k}$-mer mapping technique applied a matching threshold of at least 10 residues in orthologous viral proteins, which is of sufficient length to cover HLA-bound peptides and/or whole or part of a B cell epitope, something that is challenging using only pairwise multiple sequence alignments. Homologous peptide sequences with a BLAST bitscore indicating 10 or more residues mapped to the target sequence were recorded and parsed for display on the graph.

\section{Online SARS-CoV-2 "Immuno-analytics" resource and} analysis software

We developed an online immuno-analytics resource with an interactive plot that integrates up-to-date SARS-CoV-2 genetic variation analysis, $\mathrm{T}$ and $\mathrm{B}$ cell epitope prediction and mapping, human coronavirus homology mapping, literature meta-analysis and an accessible database for extracting data for further study. This tool is available online from http://genomics.lshtm.ac.uk/immuno. The source code for the website and up-to-date raw data files are available at https://github.com/dan-ward-bio/COVIDimmunoanalytics [18] (see Additional file 1: Fig. S1 for screenshots). The BioCircos.js library [12] was used to generate the interactive plot and Datatables.net libraries for the table. The underlying web-tool software and inhouse pipelines for data analysis are available at https:// github.com/dan-ward-bio/COVID-immunoanalytics [18].

Metadata consisting of collection date and source (geographical) location for each GISAID sequence are analysed. Temporal and geographic data on individual mutations can be found on the 'Mutation Tracker' page, accessed via the tool's home page. For the spatiotemporal mutation plots, we partitioned the whole genome sequencing dataset by week and continent and plotted non-synonymous allele frequencies using Google Charts JavaScript libraries. To improve sustainability of the tool, all functions and data of the website are generated and updated using automated data scripts developed in-house.

\section{Utility and discussion}

To demonstrate the functionality of the immunoanalytics tool, we present an analysis of the SARS-CoV-2 spike, nucleocapsid and orf3a proteins, which are vaccine and serological targets. Analysis of 150,090 SARSCoV-2 sequences identified 911,324 non-synonymous mutations across 16,951 sites in protein-coding regions; $0.71 \%$ of these mutations are singleton events and $0.03 \%$ (46) of these mutations have a frequency above $1 \%$, occurring in $>1500$ samples. The most frequent mutations were the spike protein D614G (87.3\%) and nsp12 L314P (87.5\%), which were common across all the geographical regions (all > 86\%) (Table 1), in keeping with their deep ancestral nature in the SARS-CoV-2 phylogenetic tree [24]. In particular, nsp12 L314P has been used to genotype the putative S and L strains of SARS-CoV-2, which have now been clustered into further groups [31]. Spike D614G lies 73 residues downstream from the spike $\mathrm{RBD}$, a region of interest as it is a primary target of protective humoral responses and bears immunodominant epitopes that play a possible role in antibody dependant enhancement [32-35]. We have observed a strong correlation between the spatiotemporal accumulation of both spike D614G and nsp12 L314P (Fig. 1, Table 1), 
Table 1 Most frequent non-synonymous mutations found in the 150,090 global SARS-CoV-2 whole genome sequences

\begin{tabular}{|c|c|c|c|c|c|c|c|c|c|c|c|c|}
\hline Protein* & Pos. & $\begin{array}{l}\text { Ref. } \\
\text { Allele }\end{array}$ & $\begin{array}{l}\text { Alt. } \\
\text { Allele }\end{array}$ & $\begin{array}{l}\text { Alt. Allele } \\
\text { Freq. }\end{array}$ & $\begin{array}{l}\text { Dominant Alt. Allele } \\
\text { Freq. (150090) }\end{array}$ & $\begin{array}{l}\text { Europe } \\
\text { (88266) }\end{array}$ & $\begin{array}{l}\text { Asia } \\
(7818)\end{array}$ & $\begin{array}{l}\text { NAm } \\
(38203)\end{array}$ & $\begin{array}{l}\text { SAm } \\
(1702)\end{array}$ & $\begin{array}{l}\text { AFR } \\
(1211)\end{array}$ & $\begin{array}{l}\text { OCE } \\
(12837) \\
\end{array}$ & $\begin{array}{l}\text { UK } \\
(68017)^{* *}\end{array}$ \\
\hline$S$ & 614 & D & V:G:S:N & $\begin{array}{l}1: 131490: 2: \\
10\end{array}$ & 0.877 & 0.975 & 0.940 & 0.866 & 0.890 & 0.866 & 0.868 & 0.883 \\
\hline nsp12 & 314 & $N$ & $\mathrm{H}: \mathrm{F}: \mathrm{L}: \mathrm{S}$ & $\begin{array}{l}\text { 2:114: } \\
\text { 131053:1 }\end{array}$ & 0.876 & 0.973 & 0.938 & 0.863 & 0.887 & 0.876 & 0.865 & 0.881 \\
\hline $\mathbf{N}$ & 203 & $\mathrm{R}$ & $\begin{array}{l}\text { G:K:M:l: } \\
\mathrm{S}\end{array}$ & $\begin{array}{l}\text { 9:57773:73: } \\
1: 37\end{array}$ & 0.388 & 0.426 & 0.394 & 0.374 & 0.316 & 0.393 & 0.440 & 0.380 \\
\hline $\mathbf{N}$ & 204 & G & $\begin{array}{l}R: V: L: Q: \\
T\end{array}$ & $\begin{array}{l}\text { 57,327:5: } \\
\text { 319:4:1 }\end{array}$ & 0.385 & 0.424 & 0.393 & 0.373 & 0.312 & 0.392 & 0.439 & 0.379 \\
\hline orf3a & 57 & Q & $\begin{array}{l}K: Y: R: H: \\
L\end{array}$ & $\begin{array}{l}\text { 1:6:6: } \\
\text { 35047:2 }\end{array}$ & 0.234 & 0.267 & 0.227 & 0.236 & 0.210 & 0.193 & 0.199 & 0.248 \\
\hline nsp2 & 85 & T & $V: I$ & $1: 25913$ & 0.173 & 0.195 & 0.156 & 0.180 & 0.160 & 0.090 & 0.153 & 0.180 \\
\hline nsp6 & 37 & L & $\mathrm{F}$ & 11,992 & 0.080 & 0.087 & 0.112 & 0.079 & 0.061 & 0.082 & 0.073 & 0.079 \\
\hline nsp2 & 120 & I & V:F:M & 7:11996:1 & 0.080 & 0.094 & 0.061 & 0.082 & 0.059 & 0.054 & 0.059 & 0.083 \\
\hline $\mathbf{S}$ & 222 & A & $\begin{array}{l}\mathrm{T}: \mathrm{V}: \mathrm{P}: \mathrm{I} \\
\mathrm{S}: \mathrm{F}\end{array}$ & $\begin{array}{l}\text { 4:11819:2: } \\
\text { 1:12:1 }\end{array}$ & 0.079 & 0.099 & 0.148 & 0.057 & 0.177 & 0.135 & 0.026 & 0.085 \\
\hline orf10 & 30 & V & A:I:L & 2:2:11619 & 0.078 & 0.097 & 0.146 & 0.056 & 0.176 & 0.132 & 0.025 & 0.084 \\
\hline $\mathbf{N}$ & 220 & A & $V: T$ & $11,555: 5$ & 0.077 & 0.097 & 0.146 & 0.056 & 0.176 & 0.131 & 0.025 & 0.083 \\
\hline $\mathrm{S}$ & 477 & S & $\begin{array}{l}T: R: G: I: \\
N: K\end{array}$ & $\begin{array}{l}\text { 1:19:2:59: } \\
9811: 1\end{array}$ & 0.067 & 0.077 & 0.044 & 0.070 & 0.048 & 0.045 & 0.043 & 0.068 \\
\hline N & 194 & S & $A: L: P: T$ & 30:6441:2:1 & 0.043 & 0.052 & 0.043 & 0.035 & 0.031 & 0.053 & 0.038 & 0.049 \\
\hline orf8 & 84 & L & $F: C: S: V$ & 1:1:6338:1 & 0.042 & 0.046 & 0.053 & 0.043 & 0.049 & 0.040 & 0.048 & 0.042 \\
\hline orf8 & 24 & S & L & 6151 & 0.041 & 0.053 & 0.033 & 0.036 & 0.034 & 0.009 & 0.022 & 0.048 \\
\hline $\mathrm{S}$ & 18 & L & $F: I$ & $5888: 1$ & 0.040 & 0.048 & 0.071 & 0.030 & 0.106 & 0.059 & 0.017 & 0.041 \\
\hline nsp5 & 15 & G & $S: D$ & $5433: 5$ & 0.036 & 0.039 & 0.035 & 0.036 & 0.022 & 0.038 & 0.044 & 0.036 \\
\hline orf3a & 251 & G & V:S:D:C & $5252: 31: 4: 6$ & 0.035 & 0.035 & 0.063 & 0.037 & 0.031 & 0.040 & 0.034 & 0.030 \\
\hline nsp13 & 541 & Y & C & 2606 & 0.017 & 0.019 & 0.032 & 0.017 & 0.018 & 0.021 & 0.019 & 0.017 \\
\hline nsp13 & 504 & P & L:H:S & 2535:1:111 & 0.017 & 0.020 & 0.032 & 0.017 & 0.018 & 0.022 & 0.021 & 0.017 \\
\hline
\end{tabular}

Pos. position, Freq. frequency, NAm North America, SAm South America, AFR Africa, OCE Oceania, REF reference, ALT alternative, ${ }^{*} S$ spike, $M$ membrane, $N$ nucleocapsid, ${ }^{* *}$ included in Europe

due to either a common origin and subsequently linked accumulation by a founder effect or a more complex biological interaction, including positive selection driven in part by increased transmissibility, as suggested by a recent study [36]. Specifically, the spike D614G and nsp12 N314L both appear to have a near-identical frequency with a consistent increase across all geographic regions (negating weeks with poor data collection). In contrast, the frequency of orf3a Q57H appears to fluctuate, increasing and decreasing significantly from the time it was first observed in February 2020 (week 8) to November 2020, week 43) (Fig. 1; Table 1). Using the immuno-analytical tool, spike A222V, S477N and L18F variants were observed to have increased significantly in frequency between May and November 2020 (weeks 2340). Spike mutations A222V and L18F appear to have become entrenched in Europe reaching a total frequency of $70.6 \%$ and $31.6 \%$, respectively (Additional file 1: Table S1, Additional file 1: Fig. S2). Moreover, A222V appears to be increasing in Asia and Oceania from week 41, and
S477N has increased to $>95 \%$ frequency across Oceania $(N=8321)$ with a peak of $9.3 \%$ in Europe (Additional file 1: Table S1, Additional file 1: Fig. S2), consistent with a recent report [37].

The proximity of the D614G mutation to one of the functional domains of the spike protein has raised concerns, but whether it confers any gain in pathogenicity, transmissibility or immune evasion is still unclear [32]. Other high frequency mutations occur on the nucleocapsid gene (R203K, 38.8.0\%; G204R 38.4\%; across all geographical regions $>31 \%$; Table 1 ), which has been the target antigen for several serological RDTs currently in use or in production. Both of these mutations share a near-identical spatio-temporal profile. We have identified 363 non-synonymous variant sites across the nucleocapsid gene with mutations occurring 173,955 times in this dataset. Using the SARS-CoV-2 immunoanalytics platform, we further queried these polymorphic regions for immunological relevance. The 20 residues surrounding the spike mutation D614G (S604-624) 


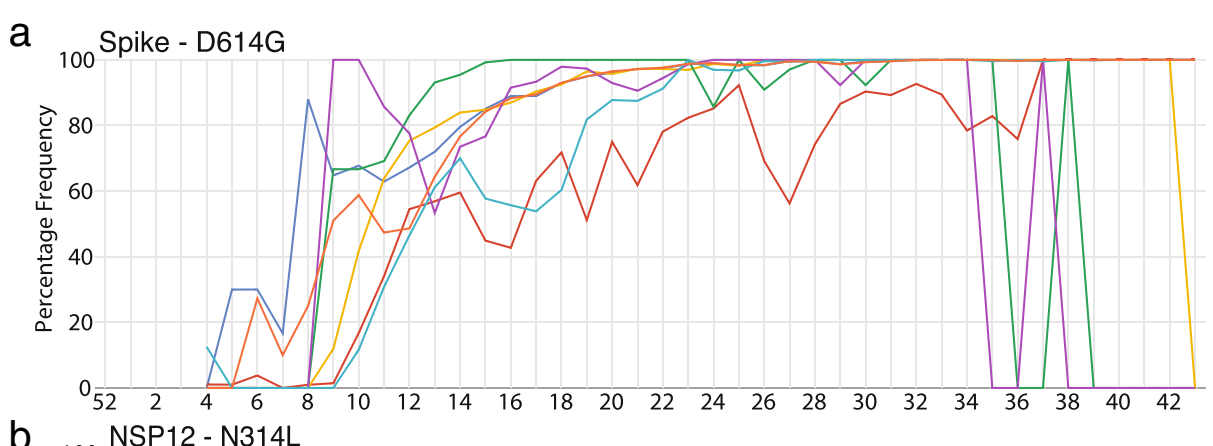

b ${ }_{100}$ NSP12-N314L

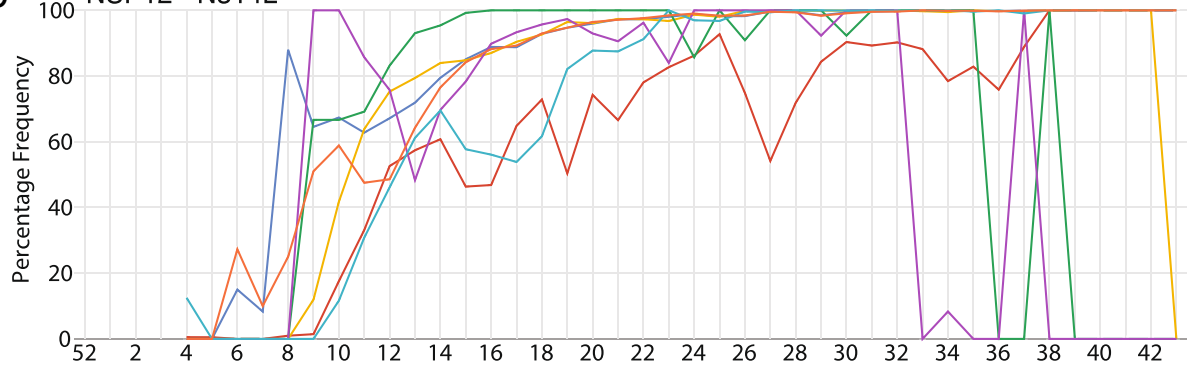

C ${ }_{100}$ ORF3a-Q57H

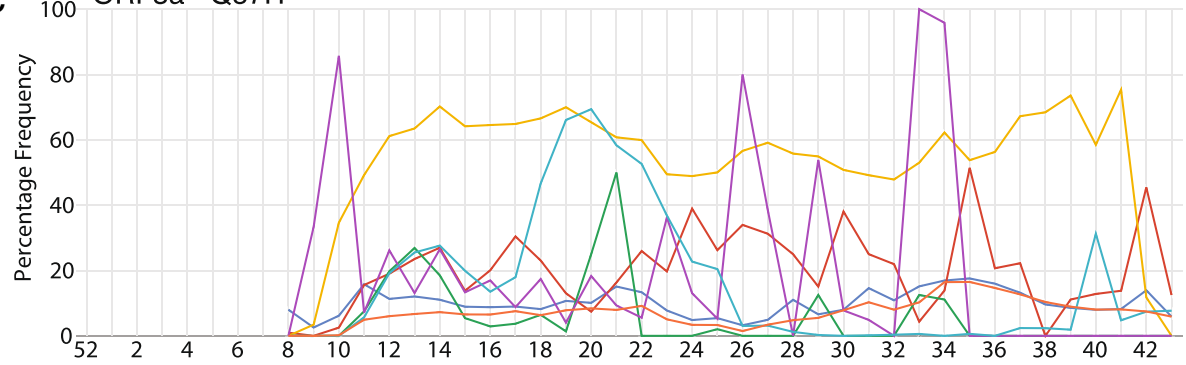

d ${ }_{14 k}$ Global Sequences by Week

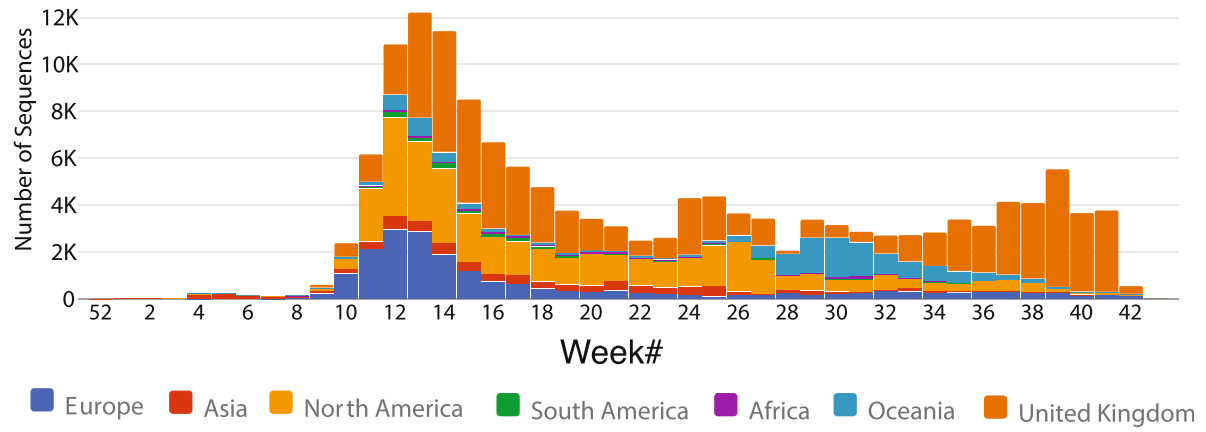

Fig. 1 a-c High frequency non-synonymous spike D614G, NSP12 N314L and orf3A Q57H mutations found in SARS-CoV-2 plotted weekly by continent. This functionality is available on the website. Users can select any mutation from the main plot and visualise it temporally and geographically. UK sequences are not included in Europe due to high frequency. $\mathbf{d}$ A stacked bar chart representing total sequences published by each continent by week. This chart is included to assist users understand how allele frequencies may be affected by poor sampling. (see http./genomics.lshtm.ac.uk/immuno/mutation_tracker)

(Fig. 2) have a high epitope prediction meta-score $(34 \%$ increase on the global median) with 204 IEDB epitope positions mapping to the surrounding residues, suggesting that this region is of high interest and may elicit a strong immune response. On top of the high level of SARS-CoV sequence homology reported, we have identified multiple clusters in the S2 domain of the spike protein, with homology to MERS, OC43, 229E, HKU1 and NL63 human coronaviruses, which may elicit a cross-reactive immune response in immune sera. $\mathrm{Hu}$ man coronavirus sequence homology is greatly reduced in the S1 domain, with only two small 10-residue pockets of OC43 and HKU1 identity (see Fig. 2). We observed a $17 \%$ increase over the median epitope meta- 


\section{SARS-CoV-2 Spike Glycoprotein}

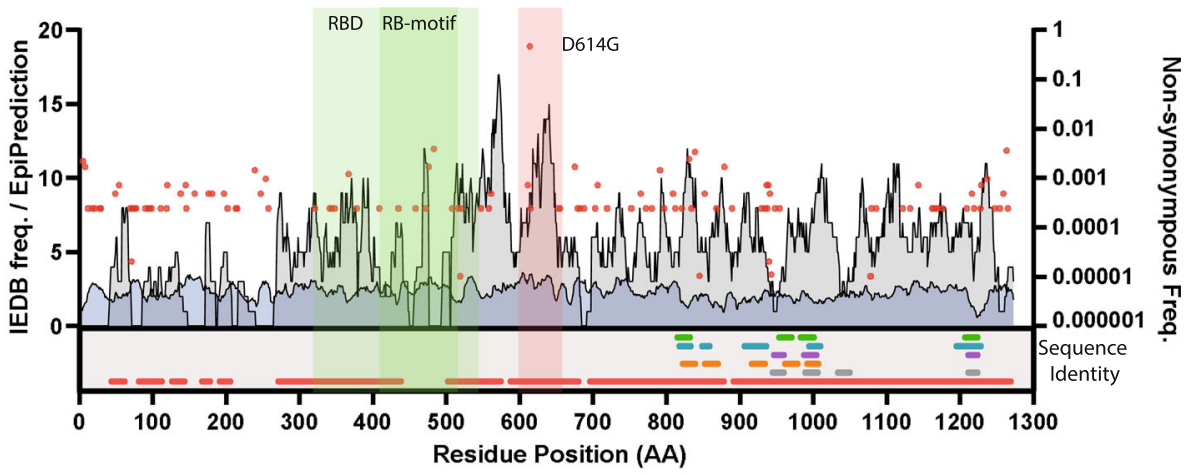

SARS-CoV-2 Nucelocapsid

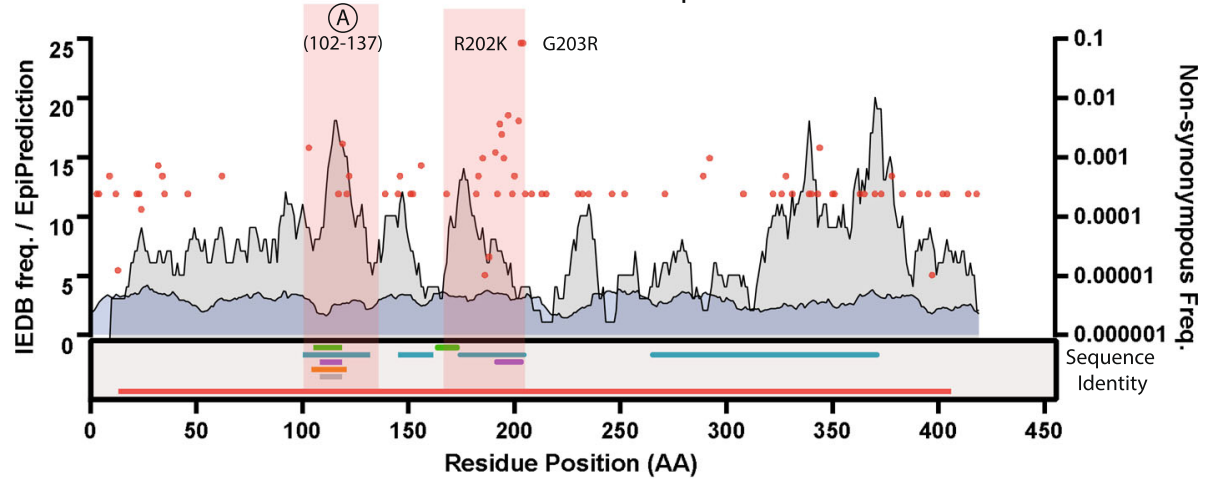

SARS-CoV-2 orf3a

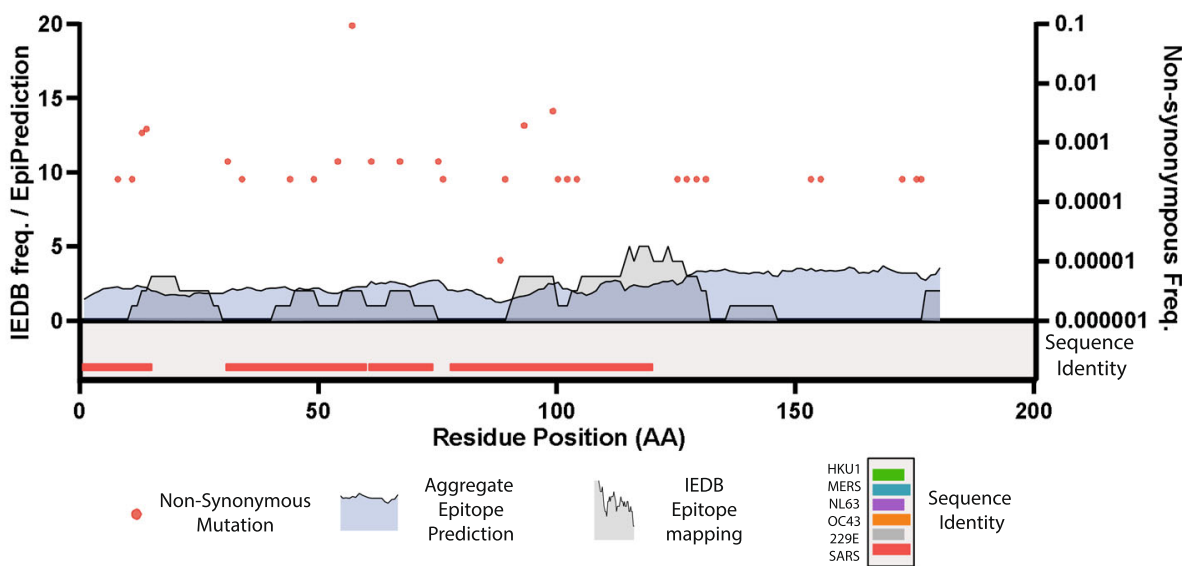

Fig. 2 Linearised extracts from the SARS-CoV-2 immuno-analytics resource database; spike, nucleocapsid and orf3a proteins. Non-synonymous mutations, B cell epitope prediction meta-score, IEDB epitope mapping and sequence identity analyses were plotted (see key). Left axis denotes the scale for epitope prediction and IEDB mapping. Right axis denotes the relative allele frequency found in the genome dataset; AA, amino acid

score within the receptor-binding motif (AA437-508), a region implicated in the direct ACE2 (angiotensin-converting enzyme 2) interaction. HLA-II peptide binding prediction (see Fig. 3) yielded several epitopes within the receptor-binding domain with high HLA-II ligand affinity, as well as strong B cell epitope prediction scores (28\% above the global median). Metadata obtained from the UniProt database reveals 3 clusters of glycosylated residues across the spike protein, a characteristic highlighted by this tool that should be considered when choosing expression systems for producing protein/peptides based on these regions.

For two high-frequency non-synonymous nucleocapsid protein mutations (R203K and G204R; co-fixed), all but three of the 30 (N173-234) flanking residues have nonsynonymous variants, with 5 sites reporting an 


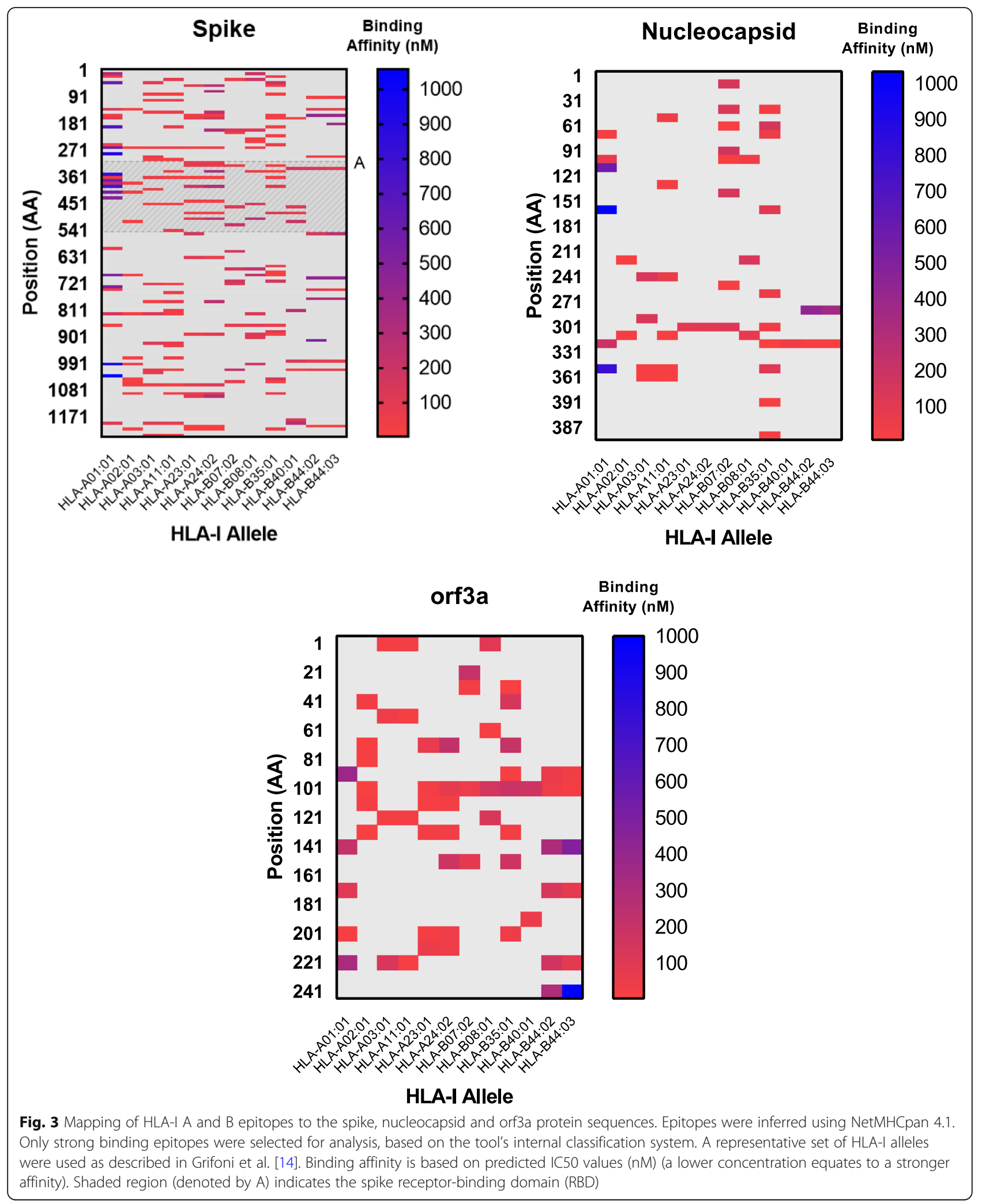


alternative allele frequency greater than 1\% (A220V, S194L, S197L, M234I and P199L:S). The average epitope meta-score for these variant sites is $30 \%$ above the global median prediction score, with the two aforementioned high frequency mutant residues scoring $35 \%$ above the global median epitope predictive score. The sequence homology analysis of the nucleocapsid protein revealed a high level of shared identity between SARS-CoV (90\%) and MERS-CoV (45\%) on a per-residue basis. The nucleocapsid protein analysis revealed two clusters of shared human coronavirus orthologue identity (Fig. 2), one of which was found to cross the aforementioned N173-234 region with identity to HKU1, NL63, MERS and SARS detected. Moreover, these clusters were found to have an increased IEDB epitope mapping frequency, high polymorphism frequency and B cell epitope metascores (23\% above the global median), indicative of potential B cell immunogenicity. We focused on two nucleocapsid protein specific regions of interest (amino acids 102 to 137 and 167 to 206; Fig. 2). Within the first 35-residue region (amino acids 102 to 137), we have detected NL63, SARS, OC43, 229E, MERS and HKU1 human coronavirus homology. Further, we observed an increase in mapped IEDB epitopes, including mapped linear peptidic $\mathrm{B}$ and $\mathrm{T}$ cell epitopes from avian gammacoronavirus, murine betacoronavirus, feline and canine alphacoronavirus- 1 providing in vitro confirmation that peptides within this region may indeed serve as immunogenic cross-reactive epitopes. The second region (amino acids 167 to 206) contains the R203K and G204R mutations along with a cluster of high frequency variants. We detected homology with HKU1, NL63 and MERS human coronavirus species along with a high frequency of SARS and murine coronavirus mapped IEDB epitopes, with a $34 \%$ increase on the median B-cell epitope prediction meta-score.

Previous studies of adaptive cellular effector immune responses to SARS-CoV infection have emphasised the importance of spike peptide presentation in the progression and severity of disease; regions of particular interest include the following: S436-443, S525-532, S366-374, S978, and S1202 [25, 26, 38]. We analysed these regions for their performance as HLA-I ligands in silico and found that all of the regions of interest had a high binding affinity scores associated with that position. Moreover, these peptides were widely represented in the predictions made across the 2915 HLA-A, $-\mathrm{B}$ and $-\mathrm{C}$ alleles used in this analysis. Taking into account all available HLA-A, B and C alleles, we found the spike peptides had an average allele coverage of $21 \%, 18 \%$ and $34 \%$ respectively. We performed an analysis to include 12 alleles with the highest frequency observed across the human population, as reported recently [14]. We found that peptides in the S366-374 and S1202 regions had high representation across the subset of 12 high frequency HLA-I alleles (Fig. 3). These findings imply that the peptides as HLA-I ligands may have a putative role in initiating a protective cellular response in SARS-CoV2 infections across a significant proportion of the HLA-I population worldwide. We have identified another region of interest that scores highly in the HLA-I peptide binding analysis. The S690-700 region has a high frequency of peptides with a high binding affinity with significant representation across all HLA-I alleles (HLAA $40 \%$, -B 23\%, -C 60\%). Furthermore, we have observed no mutations present in this region based on our SARSCoV-2 variant analysis, implying this peptide appears to remain conserved making it a prime candidate for further study. The spike D614G mutation does not appear to have significantly elevated HLA-I epitope prediction scores (Fig. 3), a finding supported by recent work [39]. The biological importance of spike D614G, particularly its immunological relevance and impact on transmission and disease, are still unclear [40, 41].

Protein 3a (orf3a) has been reported to play a role in host immune modulation by decreasing interferon alpha-receptor expression in SARS-CoV-infected cells and activating the NLRP3 inflammasome [42, 43], a response that may boost inflammation mediated COVID19 pathology. Orf3a has been a target for SARS-CoV vaccinology studies, with reports of it eliciting potentially protective responses in both protein and DNA forms $[27,44]$. These immunogenic properties appear conserved in the SARS-CoV-2 orthologue, with consistently strong antibody responses reported in COVID-19 patients [45]. Looking across the SARS-CoV-2 proteome, of the 50 residues with the highest $\mathrm{B}$ cell epitope prediction meta-score, orf3a occupies $16 \%$, despite only constituting $2.5 \%$ of the total SARS-CoV-2 protein sequence. Moreover, there are numerous high affinity HLA-II epitopes, which may serve to elicit strong antibody responses. Although protein orf3a shares a high level of identity with its SARS-CoV orthologue, we detected no amino acid sequence homology with OC43, NL63, HKU1 and 229E human coronavirus species or any nonSARS-CoV IEDB epitopes.

Our analysis of the 150,090 SAR-CoV-2 whole genome sequences detected 267 variant sites within orf3a, with non-synonymous mutations occurring 68,473 times. A minority of these variant sites are singletons $(8.2 \%)$ and five $(1.8 \%$ ) have a frequency higher than $1 \%$ (> 1500 isolates), with a non-synonymous mutation density $40 \%$ lower than that of the nucleocapsid. The variant sites identified in the orf3a gene have a mean epitope predictive meta-score of 2.3 , which is equal to the median global score, indicating that these sites may not form a part of a $\mathrm{B}$ cell epitope. Comparing the predictive metascores of the nucleocapsid protein variant sites, we 
observed an increase of $26 \%$ over the global median, indicating that nucleocapsid protein non-synonymous mutations may impact epitope variability more than those found in orf3a. $\mathrm{CD}^{+}$effector responses to protein $3 \mathrm{a}$ have been characterised in SARS-CoV patients and appear to play a significant role in immunity [26, 46, 47]. Notably, alongside two within the spike protein, a peptide in orf3a (orf3a 36-50) has been found to form a part of the public (conserved) $\mathrm{T}$ cell epitope repertoire across SARS-CoV patients [47]. This region scores highly in the HLA-II predictions with numerous HLA-A and HLA-B high affinity peptides covered (HLA-A 19\%, -B $41 \%,-C 48 \%$ ) and is relatively conserved with few low frequency non-synonymous mutations (maximum mutant allele frequency of 0.00219 (65 times)). We have identified one further region in orf3a (101-121) that scores highly with HLA-I epitope prediction across frequent HLA-I alleles (Fig. 3) and therefore may be of interest to those studying HLA-I ligands. For HLA-I prediction, we observed that orf3a performs significantly better than the nucleocapsid. Despite the nucleocapsid protein sequence being $52 \%$ larger than that of orf3a, there are $34 \%$ more high affinity HLA-I epitopes across our subset of 12 frequent HLA-I alleles (Fig. 3), which may indicate that orf3a has a more immunodominant role in cellular responses following intracellular processing when compared to the nucleocapsid protein.

Overall, we have developed an immuno-analytical tool that combines in silico prediction data with in vitro epitope mapping, SARS-CoV-2 genome variation and a kmer-based human coronavirus sequence homology with curated functional annotation data. Furthermore, we have added functionality enabling users to track mutations geographically across time. An additional framework exists to annotate positions with relevant findings from the literature to further guide users' research. The integration and co-visualisation of these data support the rational selection of diagnostics, vaccine targets with reverse immunology, and highlight regions for further immunological studies. We demonstrate the utility of the tool through the analysis of three proteins and their mutant positions, which are of relevance to current SARS-CoV-2 research.

Understanding the magnitude of transmission and patterns of infection will lead to insights for post-isolation strategies. The rapid emergence of the SARS-CoV-2 virus called for an expediated process to deploy serological RDTs for the detection of SARS-CoV-2 IgG/IgM antibody responses. There were reports early in the outbreak of lateral flow SARS-CoV-2 Ig RDTs not reaching sufficiently high levels of sensitivity and specificity [43]. While many assays use the spike protein as its sole antigen for antibody detection, others employ a combination of the spike and nucleocapsid proteins; other assays have been based solely on the nucleocapsid protein [7]. Our analyses suggest that, in its native form, the nucleocapsid protein may prove a sub-optimal target for use in serological diagnostic platforms. It possesses the greatest number of residues across all SARS-CoV-2 genes with high-frequency non-synonymous mutations, the majority of which have a high predictive epitope and IEDB epitope mapping scores when compared to variant positions of other genes. This implies that there may be an inherent variability in dominant antibody responses to different nucleocapsid protein isoforms, which may work to confound testing. We have located three regions of homology with other highly prevalent human coronavirus species, which could serve as non-specific SARS$\mathrm{CoV}-2$ epitopes if used in serological assays. Moreover, we have emphasised the high level of SARS-CoV identity across the SARS-CoV-2 proteome (except in orf8 and orf10), which may have implications for diagnostic deployment in countries that have had outbreaks involving SARS-CoV.

The spike protein has remained a focus of both vaccine and diagnostic research. Its functional role in viral entry imparts this antigen with immunodominant and neutralising antibody responses [29, 44]. This role is reinforced in our analyses, with several clusters of high epitope meta-scores in functional regions and high IEDB epitope mapping counts. The S1 domain has been the focus of a number of studies looking for specific antigens, not least because of its apparent lack of sequence homology with other human coronavirus species when compared to regions in the $\mathrm{S} 2$ domain, as well as its strong functional and immunogenic role in SARS-CoV-2 infection [7, 40, 44, 45]. However, as vaccination programmes begin, most of which will target the spike protein, it will become challenging to differentiate vaccination responses from those elicited by SARS-CoV2 infection. Therefore, alternative viable targets for serological screening may be needed.

The broad nature of the analyses performed by our tool may assist in the understanding of vaccine targets, during both design and testing phases. The prediction of HLA-I ligands is relevant not only to the study of structural viral targets, but the full range of potentially immunologically relevant endogenous proteins that may be presented following intracellular processing, some of which may have less coverage in the literature. Our broad approach to HLA-I ligand prediction ensures that researchers can assess the applicability of in silico informed vaccine targets across different populations. Further, ensuring that targets are both specific and devoid of polymorphism is essential to ensuring the longevity of vaccine responses and diagnostic capabilities, the analysis of which is achieved easily with our tool. The humoral and cellular immune responses as well as the 
affects of human coronavirus protein homology to SARS-CoV-2 proteins have yet to be fully characterised. With the significant levels of amino-acid sequence identity between SARS-CoV-2 and other human coronavirus species detected in our analysis, researchers should be wary of the potentially deleterious effects of both nonspecific humoral and cellular responses in enhancing infection; a phenomenon observed in a number of other viral pathology models. While the tracing and monitoring of non-synonymous mutations and their spatiotemporal analysis provides an initial indication of their importance, potentially the impact of evolutionary pressures on loci of interest, further analyses on signals of selection may provide additional insights. Computer intensive genome-wide analyses of positive selection are becoming available (e g. http://covid19.datamonkey.org/) and may be used to complement insights from our immuno-analytical tool.

In summary, using the SARS-CoV-2 immuno-analytics platform, we were able to identify shortcomings in current targets for diagnostics and suggest orf3a as another target for further study. This protein has proven in vitro immunogenicity in COVID-19 patients, and promising functional aspects were supported by our integrated data analysis using the tool. The database underpinning the online tool is updated automatically using data parsing scripts that require minimal human curation. The monitoring of the temporal changes in the frequencies of mutations or their presence in multiple clades in a SARS-CoV-2 phylogenetic tree could provide insights for infection control, including post-vaccine introduction. Importantly, our open-access platform and tool enables the acquisition of all of the aforementioned data associated with the SARS-CoV-2 proteome, assisting further important research on COVID-19 control tools.

\section{Conclusions}

The SARS-CoV-2 immuno-analytics platform enables the visualisation of multidimensional data to inform target selection in vaccine, diagnostic and immunological research. By integrating genomic and whole-proteome analyses with in silico epitope predictions, we have highlighted important advantages and shortcomings of two proteins at the foci of COVID-19 research (spike and nucleocapsid), while suggesting another candidate for further study (orf3a). Both spike and nucleocapsid proteins have regions of high identity shared with other endemic human coronavirus species. Moreover, several high frequency mutations found in our dataset lie within putative $\mathrm{T}$ and $\mathrm{B}$ cell epitopes, something that should be taken into consideration when designing vaccines and diagnostics. Further, our work is likely to become more important as the rollout of vaccines will introduce new selection pressures that will need to be monitored for escape variations. The immuno-analytics tool can be accessed online (http:// genomics.lshtm.ac.uk/immuno), and the source code is available on GitHub (https://github.com/dan-ward-bio/ COVID-immunoanalytics) [18].

\section{Supplementary Information}

The online version contains supplementary material available at https://doi. org/10.1186/s13073-020-00822-6.

\begin{abstract}
Additional file 1: Table S1. Extracted data from the Immuno-analytics tool. Mutations listed here have increased significantly over the 20-week period (weeks 20 to 40, year 2020) (see Fig. S2. for spike mutations A222V, S477N and L18F). Fig. S1. Screenshots from the Immuno-analytics webpage (http://genomics.Ishtm.ac.uk/immuno). Fig. S2. Screen capture from 'Mutation Tracker' page tracing spike mutations accumulating in Europe, North America and Oceania since the last week of December 2019 (week 52) into 2020 (week 1 onwards). Mutations can be traced across continents by week on the 'Mutation Tracker' page. Mutations shown here are in the Spike (A222V, S477N and L18F).
\end{abstract}

\section{Abbreviations}

COVID-19: Coronavirus disease (2019); SARS-CoV-2: Severe acute respiratory syndrome-coronavirus (2); RT-qPCR: Reverse transcriptase-quantitative polymerase chain reaction; RNA: Ribonucleic acid; RDT: Rapid diagnostic test; ELISA: Enzyme-linked immunosorbent assay; RBD: Receptor-binding domain; HLA-I and HLA-II: Human leukocyte antigen; IEDB: Immune epitope database; SARS: Severe acute respiratory syndrome; MERS: Middle eastern respiratory syndrome; GFF: General feature format; ORF: Open reading frame; CLI: Command line interface; CD8: Cluster of differentiation (8);

ACE2: Angiotensin-converting enzyme 2; NLRP3: NLR family pyrin domain containing 3; IC50: 50\% inhibitory concentration

\section{Acknowledgements}

We gratefully acknowledge the laboratories who submitted the data to the IEDB, NCBI and GISAID public databases on which this research is based. We also thank IEDB, NCBI and GISAID for developing and curating their databases. We gratefully acknowledge the availability of the Medical Research Council UK funded eMedLab (HDR UK) computing resource.

\section{Authors' contributions}

DW, SC and TGC conceived and directed the project. MH and JEP provided software and informatic support. DW and JEP performed bioinformatic and statistical analyses under the supervision of TGC. DW, MLH, SC and TGC interpreted results. DW wrote the first draft of the manuscript. All authors commented and edited on various versions of the draft manuscript. DW, TGC and SC compiled the final manuscript. All authors read and approved the final manuscript.

\section{Funding}

DW is funded by a Bloomsbury Research PhD studentship. SC is funded by Bloomsbury SET, Medical Research Council UK (MR/M01360X/1, MR/R025576/ 1 and MR/R020973/1) and BBSRC UK (Grant no. BB/R013063/1) grants. TGC is funded by the Medical Research Council UK (Grant no. MR/M01360X/1, MR/ N010469/1, MR/R025576/1 and MR/R020973/1) and BBSRC UK (Grant no. BB/ $\mathrm{R} 013063 / 1)$.

\section{Availability of data and materials}

The sequencing data analysed during the current study are available from GISAID (https://www.gisaid.org) and NCBI (https://www.ncbi.nlm.nih.gov). Full analysis datasets can be downloaded from http://genomics.Ishtm.ac.uk/ immuno or https://github.com/dan-ward-bio/COVID-immunoanalytics [18]. The source code for the website can be accessed through https://github. com/dan-ward-bio/COVID-immunoanalytics [18].

Ethics approval and consent to participate Not applicable 


\section{Consent for publication}

Not applicable

\section{Competing interests}

The authors declare that they have no competing interests.

\section{Author details}

'Department of Infection Biology, Faculty of Infectious and Tropical Diseases, London School of Hygiene and Tropical Medicine, Keppel Street, London WC1E 7HT, UK. ${ }^{2}$ Faculty of Epidemiology and Population Health, London School of Hygiene and Tropical Medicine, Keppel Street, London WC1E 7HT, UK.

Received: 12 May 2020 Accepted: 14 December 2020 Published online: 07 January 2021

\section{References}

1. IMF. World Economic Outlook Update, June 2020: A Crisis Like No Other, An Uncertain Recovery [Internet]. IMF. 2020. [cited 2020 Nov 10]. Available from: https://www.imf.org/en/Publications/WEO/Issues/2020/06/24/ WEOUpdateJune2020.

2. Dong $\mathrm{E}, \mathrm{Du} \mathrm{H}$, Gardner L. An interactive web-based dashboard to track COVID-19 in real time. Lancet Infect. Dis. Elsevier; 2020. p. 533-4. https://doi. org/10.1016/S1473-3099(20)30120-1

3. Verity R, Okell LC, Dorigatti I, Winskill P, Whittaker C, Imai N, et al. Estimates of the severity of coronavirus disease 2019 : a model-based analysis. Lancet Infect Dis. 2020;3099:1-9.

4. Vogels CBF, Brito AF, Wyllie AL, Fauver JR, Ott IM, Kalinich CC, et al. Analytical sensitivity and efficiency comparisons of SARS-COV-2 qRT-PCR assays. medRxiv; 2020;2020.03.30.20048108.

5. CDC. Processing of sputum specimens for nucleic acid extraction. 2020

6. Long Q-X, Liu B-Z, Deng H-J, Wu G-C, Deng K, Chen Y-K, et al. Antibody responses to SARS-CoV-2 in patients with COVID-19. Nat Med. 2020;26:845-8.

7. JHU Centre for Health Security: Global Progress on COVID-19 SerologyBased Testing. http://www.centerforhealthsecurity.org/resources/COVID-19/ Serology-based-tests-for-COVID-19.html\#sec1. [Accessed 3 Apr 2020].

8. GeurtsvanKessel CH, Okba NMA, Igloi Z, Bogers S, Embregts CWE, Laksono BM, et al. An evaluation of COVID-19 serological assays informs future diagnostics and exposure assessment. Nat Commun. 2020;11:3436. Available from: https://doi.org/10.1038/s41467-020-17317-y.

9. World Health Organisation. Landscape of COVID-19 candidate vaccines [Internet]. https://www.who.int/blueprint/priority-diseases/key-action/novelcoronavirus-landscape-ncov.pdf?ua=1. [Accessed 3 Apr 2020].

10. Thanh Le T, Andreadakis Z, Kumar A, Gómez Román R, Tollefsen S, Saville M, et al. The COVD-19 vaccine development landscape. Nat Rev Drug Discov. https:/doi. org/1038/d41573-020-00073-5.

11. Parker EPK, Shrotri M, Kampmann B. Keeping track of the SARS-CoV-2 vaccine pipeline. Nat Rev Immunol. 2020;20:650

12. Cui Y, Chen X, Luo H, Fan Z, Luo J, He S, et al. BioCircos.js: an interactive Circos JavaScript library for biological data visualization on web applications. Bioinformatics. 2016;32:1740-2.

13. Vita R, Mahajan S, Overton JA, Dhanda SK, Martini S, Cantrell JR, et al. The Immune Epitope Database (IEDB): 2018 update. Nucleic Acids Res. 2019;47: D339-43.

14. Grifoni A, Sidney J, Zhang Y, Scheuermann RH, Peters B, Sette A. A sequence homology and bioinformatic approach can predict candidate targets for immune responses to SARS-CoV-2. Cell Host Microbe. 2020;27: 671-80 e2.

15. Katoh K, Standley DM. MAFFT multiple sequence alignment software version 7: improvements in performance and usability. Mol Biol Evol. 2013; 30:772-80.

16. Quinlan AR, Hall IM. BEDTools: a flexible suite of utilities for comparing genomic features. Bioinformatics. 2010;26:841-2

17. Rice P, Longden L, Bleasby A. EMBOSS: The European Molecular Biology Open Software Suite. Trends Genet. Elsevier Ltd; 2000. p. 276-7. https://doi org/10.1016/s0168-9525(00)02024-2.

18. COVID Immunoanalytics GitHub Page. https://github.com/dan-ward-bio/ COVID-immunoanalytics. Accessed 1 Dec 2020.

19. Jespersen MC, Peters B, Nielsen M, Marcatili P. BepiPred-2.0: improving sequence-based B-cell epitope prediction using conformational epitopes. Nucleic Acids Res. 2017;45:W24-9.
20. Davydov YI, Tonevitsky AG. Prediction of linear B-cell epitopes. Mol Biol Springer. 2009:43:150-8.

21. Sher G, Zhi D, Zhang S. DRREP: deep ridge regressed epitope predictor. BMC Genomics. 2017;18:676.

22. Saha S, Raghava GPS. Prediction of continuous B-cell epitopes in an antigen using recurrent neural network. Proteins Struct Funct Bioinforma. 2006;65: 40-8.

23. Singh $H$, Ansari HR, Raghava GPS. Improved method for linear B-cell epitope prediction using antigen's primary sequence. Schönbach C, editor. PLoS One; 2013;8:e62216.

24. Saha S, Raghava GPS. ICARIS 2004, LNCS 3239; 2004

25. Zhi Y, Kobinger GP, Jordan H, Suchma K, Weiss SR, Shen $H$, et al. Identification of murine CD8 T cell epitopes in codon-optimized SARSassociated coronavirus spike protein. Virology. 2005;335:34-45.

26. Channappanavar R, Fett C, Zhao J, Meyerholz DK, Perlman S. Virus-specific memory CD8 T cells provide substantial protection from lethal severe acute respiratory syndrome coronavirus infection. J Virol. 2014:88:11034-44.

27. Lu B, Tao L, Wang T, Zheng Z, Li B, Chen Z, et al. Humoral and cellular immune responses induced by 3 a DNA vaccines against severe acute respiratory syndrome (SARS) or SARS-like coronavirus in mice. Clin Vaccine Immunol. 2009;16:73-7.

28. Jurtz V, Paul S, Andreatta M, Marcatili P, Peters B, Nielsen M. NetMHCpan-4.0: improved peptide-MHC class I interaction predictions integrating eluted ligand and peptide binding affinity data. J Immunol. 2017;199:3360-8.

29. Chen B, Khodadoust MS, Olsson N, Wagar LE, Fast E, Liu CL, et al. Predicting HLA class II antigen presentation through integrated deep learning. Nat Biotechnol. 2019;37:1332-43.

30. Altschul SF, Gish W, Miller W, Myers EW, Lipman DJ. Basic local alignment search tool. J Mol Biol. 1990;215:403-10.

31. Phelan J, Deelder W, Ward D, Campino S, Hibberd ML, Clark TG. Controlling the SARS-CoV-2 outbreak, insights from large scale whole genome sequences generated across the world. bioRxiv; 2020;2020.04.28.066977.

32. Sui J, Deming M, Rockx B, Liddington RC, Zhu QK, Baric RS, et al. Effects of human anti-spike protein receptor binding domain antibodies on severe acute respiratory syndrome coronavirus neutralization escape and fitness. J Virol. 2014:88:13769-80.

33. Wang SF, Tseng SP, Yen CH, Yang JY, Tsao CH, Shen CW, et al. Antibodydependent SARS coronavirus infection is mediated by antibodies against spike proteins. Biochem Biophys Res Commun. 2014;451:208-14.

34. Korber B, Fischer W, Gnanakaran SG, Yoon H, Theiler J, Abfalterer W, et al. Spike mutation pipeline reveals the emergence of a more transmissible form of SARS-CoV-2. bioRxiv; 2020;2020.04.29.069054.

35. Huang AT, Garcia-Carreras B, Hitchings MDT, Yang B, Katzelnick LC, Rattigan SM, et al. A systematic review of antibody mediated immunity to coronaviruses: kinetics, correlates of protection, and association with severity. Nat Commun. 2020;11:4704.

36. Korber B, Fischer WM, Gnanakaran S, Yoon H, Theiler J, Abfalterer W, et al. Tracking changes in SARS-CoV-2 spike: evidence that D614G increases infectivity of the COVID-19 virus. Cell. 2020;182:812-27 e19.

37. Hodcroft EB, Zuber M, Nadeau S, Comas I, González Candelas F, Consortium S-S, et al. Emergence and spread of a SARS-CoV-2 variant through Europe in the summer of 2020. medRxiv; 2020;2020.10.25.20219063.

38. Chen $H_{1}$ Hou J, Jiang $X$, Ma S, Meng M, Wang $B$, et al. Response of memory CD8 $+\mathrm{T}$ cells to severe acute respiratory syndrome (SARS) coronavirus in recovered SARS patients and healthy individuals. J Immunol. 2005;175:591-8

39. Kiyotani $K$, Toyoshima $Y$, Nemoto $K$, Nakamura $Y$. Bioinformatic prediction of potential T cell epitopes for SARS-Cov-2. J Hum Genet. 2020;65:569-75.

40. Grubaugh ND, Hanage WP, Rasmussen AL. Leading edge making sense of mutation: what D614G means for the COVID-19 pandemic remains unclear; 2020

41. Volz EM, Hill V, McCrone JT, Price A, Jorgensen D, O\&\#039; Toole A, et al. Evaluating the effects of SARS-CoV-2 Spike mutation D614G on transmissibility and pathogenicity. medRxiv. 2020;2020.07.31.20166082.

42. Minakshi R, Padhan K, Rani M, Khan N, Ahmad F, Jameel S. The SARS coronavirus 3 a protein causes endoplasmic reticulum stress and induces ligand-independent downregulation of the type 1 interferon receptor. PLoS One. 2009;4(12):e8342. https://doi.org/10.1371/journal.pone.0008342.

43. Siu KL, Yuen KS, Castano-Rodriquez C, Ye ZW, Yeung ML, Fung SY, et al. Severe acute respiratory syndrome coronavirus ORF3a protein activates the NLRP3 inflammasome by promoting TRAF3-dependent ubiquitination of ASC. FASEB J. 2019;33:8865-77. 
44. Zhong $X$, Guo Z, Yang H, Peng L, Xie Y, Wong TY, et al. Amino terminus of the SARS coronavirus protein 3a elicits strong, potentially protective humoral responses in infected patients. J Gen Virol. 2006;87:369-74.

45. Wang $H$, Hou X, Wu X, Liang T, Zhang X, Wang D, et al. SARS-CoV-2 proteome microarray for mapping COVID-19 antibody interactions at amino acid resolution. bioRxiv. 2020;2020(03):26.994756.

46. Oh H-LJ, Chia A, Chang CXL, Leong HN, Ling KL, Grotenbreg GM, et al. Engineering $T$ cells specific for a dominant severe acute respiratory syndrome coronavirus CD8 T cell epitope. J Virol. 2011;85:10464-71.

47. Li CK, Wu H, Yan H, Ma S, Wang L, Zhang M, et al. T cell responses to whole SARS coronavirus in humans. J Immunol. 2008;181:5490-500.

\section{Publisher's Note}

Springer Nature remains neutral with regard to jurisdictional claims in published maps and institutional affiliations.

Ready to submit your research? Choose BMC and benefit from:

- fast, convenient online submission

- thorough peer review by experienced researchers in your field

- rapid publication on acceptance

- support for research data, including large and complex data types

- gold Open Access which fosters wider collaboration and increased citations

- maximum visibility for your research: over $100 \mathrm{M}$ website views per year

At BMC, research is always in progress.

Learn more biomedcentral.com/submissions 